\title{
Notification of tuberculosis: how many cases are never reported?
}

\author{
C D Sheldon, K King, H Cock, P Wilkinson, N C Barnes
}

\begin{abstract}
Background Notification of tuberculosis is essential for local contact tracing and for assessing the national incidence of tuberculosis. The accuracy of notification figures is uncertain. This study examined the notification rates of all patients diagnosed as having tuberculosis at two hospitals in the East End of London over five years.

Methods In a retrospective survey of all patients aged 16 years or more presenting with tuberculosis to the London Chest Hospital or the Royal London Hospital from 1 January 1985 to 31 December 1989 , cases of tuberculosis were identified from microbiology and histology records, satutory notifications, necropsy reports, coroners' records, hospital activity data, and death certificates. Clinical data were obtained from case notes and notification was determined from the local authority notification lists.
\end{abstract}

Results Six hundred and nine adult patients with tuberculosis were identified. Notes were available for 580 cases $(95 \%)$, of which $426(73 \%)$ had been notified. The proportion of cases notified varied according to the specialty of the clinician in charge of the patient at diagnosis. Patients with a past history of tuberculosis and those who died within one year were less likely to have had their tuberculosis notified. Age, race, and lack of microbial or histological confirmation of diagnosis did not influence the proportion of cases notified. One hundred and eighty five patients had smear positive sputum, but 25 of these cases (14\%) were not notified. Eighty five patients who had presented with pulmonary tuberculosis did not have their disease notified; 20 (24\%) had smear positive sputum.

Conclusions Many cases of tuberculosis are not notified (27\%). Fourteen per cent of all sputum smear positive cases of tuberculosis were not notified, and these patients are a considerable public health risk. The true incidence of tuberculosis in the area studied is at least one third higher than current notification figures suggest.

(Thorax 1992;47:1015-1018)

Tuberculosis is a notifiable disease but the value of the notification system depends on complete reporting of cases. Notifications contribute to the control of tuberculosis through appropriate contact tracing, which has two main functions: identifying people whom the patient may have infected and finding the person who infected the patient. Notifications of tuberculosis also provide statistics of incidence on which control measures, such as the BCG programme, can be based. In England and Wales there is a statutory requirement that doctors should notify all cases of tuberculosis to the "proper officer" of the appropriate borough, who is usually the consultant in communicable disease control. A recent survey of one local health authority, however, showed that a third of doctors did not know that tuberculosis was notifiable and $70 \%$ did not know where to find a notification form. ${ }^{1}$ Most European and North American countries require clinicians to notify cases of tuberculosis to a responsible authority, whose role in the control of tuberculosis is defined by legal stipulations.

There is limited information on the accuracy of notification figures in England and Wales, ${ }^{2}$ but a study in Scotland ${ }^{3}$ found that $40 \%$ of cases with a combined clinical and pathological diagnosis were not notified. A similar proportion of cases was unreported in the United States. ${ }^{45}$ Many cases of notifiable diseases other than tuberculosis are not notified even during outbreaks of disease, ${ }^{67}$ and a survey of doctors in one health district showed that there is considerable uncertainty about which infectious diseases are notifiable. ${ }^{8}$

The Royal London Group of hospitals and the London Chest Hospital are situated in the East End of London, an area with a high unemployment rate and a large immigrant population. Most patients come from the boroughs of Tower Hamlets and Hackney, which have tuberculosis notification rates of 30 $50 / 100000$ population, compared with a national average of $10 / 100000$ in $1987 .^{9}$ Patients with tuberculosis may present to doctors in a wide range of specialties. This study examined the extent of underreporting among adult patients with tuberculosis in this area of East London with a high incidence of tuberculosis and attempted to identify factors that may have contributed to a failure of notification.

\section{Patients and methods}

All patients with tuberculosis aged 16 years or more who attented the London Chest Hospital or Royal London Group of Hospitals from 1 January 1985 to 31 December 1989 were identified. Possible cases of tuberculosis were identified from the following sources: micro- 


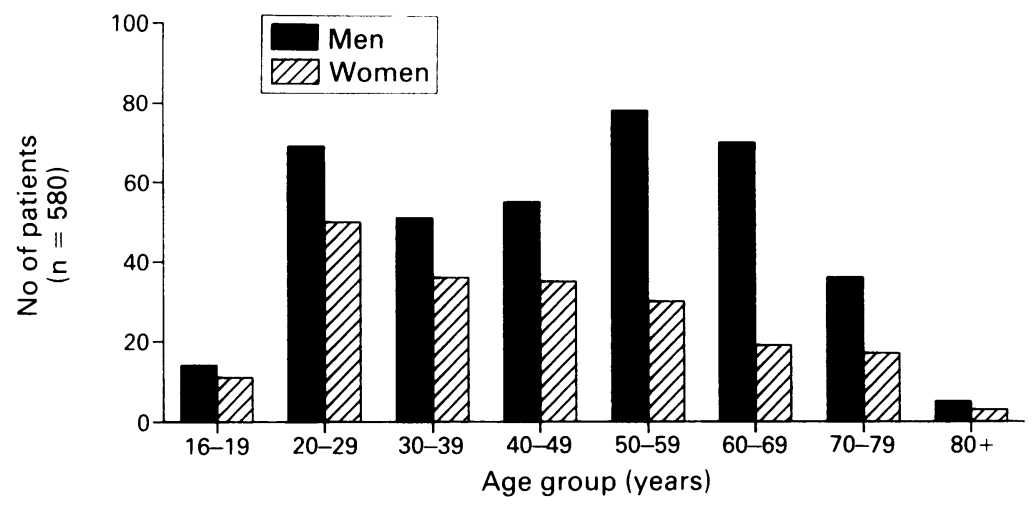

Age and sex distribution of all 580 patients with tuberculosis.

biological reports of acid fast rods or Mycobacterium tuberculosis; histological records that reported granulomas, caseation, or features diagnostic of tuberculosis; statutory notifications; necropsy and coroners' reports; death certificates; and hospital activity data coded by diagnosis. Definitive histological evidence of tuberculosis was considered to be the presence of two of the following features: acid fast bacilli seen in the sample, caseating necrosis, granulomas. Case notes of patients identified from these sources were examined and only those patients diagnosed as having tuberculosis were included in the study.

Information on whether cases had been notified was obtained by examining all the notifications received by the local authorities during the study period and the following six months.

Clinical information was collected on a standard form and analysed by using the epidemiological program Epi Info. Proportions were compared by means of the $\chi^{2}$ test.

The study was approved by the medical committees of the London Chest Hospital and the Royal London Hospital.

\section{Results}

Six hundred and nine patients had active tuberculosis diagnosed during the study period. Notes were available for $580(95 \%)$. The results therefore relate to the 580 patients for whom clinical information was available.

The age and sex distribution of patients is shown in the figure and the ethnic origin of patients in table 1 .

Four hundred and twenty six of the cases of tuberculosis $(73 \%)$ were notified; no evidence of notification was found for the remaining 154 patients $(27 \%)$.

Samples of sputum from 185 patients showed acid fast bacilli but 25 of these cases

Table 1 Ethnic group of patients with tuberculosis

\begin{tabular}{lc}
\hline Ethnic group & No $(\%)$ of cases \\
\hline Indian Subcontinent & $264(45)$ \\
White & $236(41)$ \\
African & $15(3)$ \\
West Indian & $12(2)$ \\
Chinese & $13(2)$ \\
Other & $40(7)$ \\
$\quad$ Total & $580(100)$ \\
\hline
\end{tabular}

$(14 \%)$ had not been notified. Eighty five patients who had presented with pulmonary tuberculosis had not had their disease notified; 20 $(24 \%)$ had smear positive sputum. Cases in which acid fast bacilli were seen in any pathological sample were more likely to be notified (table 2). Notification rates were similar, however, for cases confirmed by culture (265/ $354,75 \%$ ) and those not confirmed by culture $\left(161 / 226,71 \%, \chi^{2}=0.93,1 \mathrm{df} ; \mathrm{p}=0.335\right)$. Notification rates were similar for those patients with laboratory support for the diagnosis of tuberculosis and for those with a clinical diagnosis alone. Four hundred and thirty nine patients had a diagnosis of tuberculosis based on either a positive culture of Mycobacterium tuberculosis, a positive acid fast smear, or a definitive histological sample. The remaining 141 patients had a clinical diagnosis of tuberculosis based on clinical symptoms, signs, and characteristic radiological features but without definitive microbiological or histological confirmation. Three hundred and twenty five (74\%) of the cases with a confirmed diagnosed of tuberculosis were notified, compared with 101 $(72 \%)$ of the cases with a clinical diagnosis alone.

Two hundred and two patients had histological samples submitted as part of their diagnostic procedures. Seventy of 111 cases with definitive histological samples were notified (63\%) compared with 58 of 91 (64\%) cases with histological appearances not diagnostic of tuberculosis. Nine patients were diagnosed at necropsy as having active tuberculosis that substantially contributed to, or caused, the death of the patient.

The specialty of the clinician in charge of the patient at the time of diagnosis of tuberculosis is shown in table 3. The percentage of cases notified varied from $17 \%$ to $83 \%$. Those clinicians who diagnosed the most cases of tuberculosis also notified the largest proportion of cases. Chest physicians were more likely to notify cases of tuberculosis than clinicians of all other specialties combined $\left(\chi^{2}=37.59,1 \mathrm{df}\right.$; $\mathrm{p}<0.0001$.) Chest physicians saw the greatest number of cases (377) but they failed to notify $69(18 \%)$ of these, which represent $45 \%$ of the total number not notified.

A history of tuberculosis at least one year before presentation was recorded in 106 patients. The time from past tuberculosis to current presentation was known for 96 patients and was more than two years for 90 patients; the median time from last diagnosis of tuberculosis was 10.5 years. Four hundred and thirty eight patients had no history of previous disease; 36 patients had an inadequate history recorded in the notes. Sixty six $(63 \%)$ of those with a past history had their tuberculosis notified compared with 339 of $434(78 \%)$ with no history of tuberculosis $\left(\chi^{2}=10.53,1 \mathrm{df}\right.$; $\mathrm{p}=0.001$ ).

Fifty one patients died within six months of the diagnosis of tuberculosis (six died before the diagnosis was made and only two of these cases were notified). Those patients who died within six months or before the diagnosis of tuberculosis had been made were considerably 
Table 2 Notifications of tuberculosis in patients with acid fast bacilli seen in any pathological sample

\begin{tabular}{lrrr}
\hline & \multicolumn{2}{l}{ No (\%) of cases } & \\
\cline { 2 - 3 } & Notified & Not notified & Total \\
\hline Smear positive with any pathological sample & $205(84)$ & $38(16)$ & 243 \\
Smear negative & $195(64)$ & $110(36)$ & 305 \\
No sample sent for smear & $26(81)$ & $6(19)$ & 32 \\
\hline
\end{tabular}

$\chi^{2}=30 \cdot 00,2 \mathrm{df} ; \mathrm{p}=<0.00001$.

less likely to have the disease notified ( 26 of 51 patients) than those who survived (400 of 529 surviving patients: $\chi^{2}=16 \cdot 8,1 \mathrm{df}$; $\mathrm{p}=0.00005$ ). Death was significantly associated with the presence of any coexisting disease. Forty two $(\mathbf{8 2} \%)$ of those who died had one or more coexisting illnesses (malignancy $48 \%$, chronic airflow obstruction $29 \%$, diabetes $17 \%$, renal failure $12 \%$, other serious disease $38 \%$ ). Only $40 \%$ of those who survived more than six months had any associated medical condition at presentation $\left(\chi^{2}=34 \cdot 78,1 \mathrm{df}\right.$; $\mathrm{p}=<0.00001)$. Only four patients in the entire series were known to have HIV infection; two of these cases of tuberculosis were notified.

\section{Discussion}

Tuberculosis, whether infectious or not, is a notifiable disease. This survey has shown substantial underreporting, a finding that is important for contacts of those patients with tuberculosis and also for national statistiscs.

This study has examined the extent of undernotification of tuberculosis in a different way from previous studies in that it reports the notification rate for all adults diagnosed as suffering from tuberculosis in two hospitals over five years. An extensive survey of tuberculosis notifications in England and Wales over six months examined the accuracy of data reported by medical officers for environmental health to the Office of Population Censuses and Surveys by comparision with notification forms completed by chest physicians. ${ }^{2}$ Although certain inaccuracies in the reporting of tuberculosis were noted, the extent of any underreporting could not be measured. A survey of clinical and pathological diagnoses of tuberculosis, which found that almost $40 \%$ of cases were not notified, was confined to one university department in Scotland, where there are

Table 3 Notifications of tuberculosis by specialty of the consultant in charge of the patient at the time of diagnosis

\begin{tabular}{llcc}
\hline & \multicolumn{2}{l}{ No of cases } & \\
\cline { 2 - 3 } Specialty & Seen & Notified & \\
\hline Chest medicine & 377 & 308 & 82 \\
General medicine & 94 & 64 & 68 \\
Surgery & 39 & 24 & 62 \\
Neurology or neurosurgery & 18 & 9 & 50 \\
Renal medicine & 12 & 2 & 17 \\
Haematology & 6 & 1 & 17 \\
Others & 34 & 18 & 53 \\
$\quad$ Total & 580 & 426 & 73 \\
\hline
\end{tabular}

different procedures for the notification of tuberculosis, including notification by microbiologists. $^{3}$

The likelihood of tuberculosis in contacts of patients with tuberculosis varies with the type of contact, the site of disease, and the ethnic group of the index case. Household contacts of a person with smear positive disease are at most risk, $9 \%$ of Asians and $12 \%$ of non-Asians developing active tuberculosis. ${ }^{10}$ The risk declines to $1 \%$ among close contacts of those with non-pulmonary disease and to $0.3 \%$ among casual contacts of those with smear positive disease, such as work colleagues. These figures may be higher in some communities. ${ }^{11}$ There are clearly established guidelines for the investigation of contacts of patients with tuberculosis ${ }^{12}$ but these cannot address the problem of unnotified cases. The level of underreporting and the known incidence of tuberculosis in contacts suggests that a substantial number of our patients' contacts may have active tuberculosis. This population could contribute to the high prevalence of tuberculosis in this area.

The wide variation in the percentage of cases notified among the different specialties of the consultant in charge at the time of diagnosis (table 3) suggests that the more cases of tuberculosis a team sees the more likely the case is to be notified. Chest physicians appear more likely to notify cases than other specialists, and this may be a further reason for all patients with tuberculosis to be reviewed by a chest physician, as has been suggested. ${ }^{13}$ The number of notifications recorded for surgeons may be artificially high because after an initial diagnosis of tuberculosis most of their patients were subsequently referred to chest physicians for further management and cases may have been notified on the advice of the chest physicians. If the tendency for those who see fewer cases of tuberculosis to notify fewer cases holds for the rest of England and Wales, national figures for areas with low notification rates may seriously underestimate the true incidence of tuberculosis. The age, sex, and ethnic origin of patients in this study closely parallel the reported population characteristics of all patients with tuberculosis in England and Wales. ${ }^{914}$ During 1987 , the mid point of this study, the nationally notified number of cases of tuberculosis was $5085,{ }^{9}$ but if the extent of undernotification reported here is reproduced throughout England and Wales then the true figure would be about 1800 more.

Patients with a past history of tuberculosis and those who died within six months of diagnosis were less likely to have their disease notified. We cannot be sure of the reason for this but many doctors apparently think that tuberculosis in a patient who has already had the disease (and who may have had it notified in the past) does not need to be notified again. Similarly, many of the patients who died within six months of diagnosis had coexisting diseases, and these, rather than tuberculosis, may have been seen as the major problem.

There was considerable undernotification of smear positive pulmonary tuberculosis, $14 \%$ of 
sputum smear positive cases not being notified. The lack of notification in so many patients represents a substantial potential public health risk. In an attempt to try to increase the number of notifications, and in the light of published proposals by others, we have suggested some local measures that could be applied more widely. These include notification of all positive cultures by microbiologists (as in Scotland), notification by histopathologists of likely cases, ${ }^{3}$ notification by pharmacists of all patients prescribed antituberculosis drugs, ${ }^{15}$ attention to specialties with low notification rates, and education of hospital staff about tuberculosis and the offer by chest physicians to see all patients with tuberculosis for advice on treatment and further management.

1 Harvey I. Infectious disease notification-a neglected legal requirement. Health Trends 1991;23:73-4.

2 Davies PD, Darbyshire JH, Nunn AJ, Byfield SP, Fox W, Citron KM, et al. Ambiguities and inaccuracies in the notification system for tuberculosis in England and Wales. notification system for tuberculosis

3 Bradley BL, Kerr KM, Leitch AG, Lamb D. Notification of tuberculosis: can the pathologist help? $B M J 1988$ 297:595.
4 Marier R. The reporting of communicable diseases. Am J Epidemiol 1977;105:587-90.

5 Tizes R, Hayden C, Tizes CW. The source of notification in tuberculosis. Am J Public Health 1974;64:809-11.

6 Jenkinson D. Whooping cough: what proportion of cases is notified in an epidemic? $B M J 1983 ; 287: 185-6$.

7 Cartwright KAV, Stuart JM, Noah ND. An outbreak of meningococcal disease in Gloucestershire. Lancet 1986; ii:558-61.

8 Voss S. How much do doctors know about the notification of infectious diseases? BMJ 1992;304:755.

9 Office of Population Censuses and Surveys. Communicable disease statistics: statistical tables, 1987. London: HMSO, 1989. (Series MB2 No 14).

10 British Thoracic and Tuberculosis Association. A study of standardized contact procedure in tuberculosis. Tubercle 1978;59:245-59.

11 Capewell S, Leitch AG. The value of contact procedures for tuberculosis in Edinburgh. Br J Dis Chest 1984;78: 317-29.

12 Subcommittee of the Joint Tuberculosis Committee of the British Thoracic Society. Control and prevention of tuberculosis in Britain: an updated code of practice. $B M J$ 1990;300:995-9.

13 Ormerod LP. Chemotherapy and management of tuberculosis in the United Kingdom: recommendations of the culosis in the United Kingdom: recommendations of the Joint Tuberculosis Committee
Society. Thorax 1990;45:403-8.

14 Nunn AJ, Darbyshire JH, Fox W, Johnson DA, Springett VH. Changes in annual tuberculosis notification rates between 1978/79 and 1983 for the population of Indian subcontinent ethnic origin resident in England. J Epidemiol Community Health 1986;40:357-63.

15 Shanks NJ, Lambourne A, Kuhaymi RA, Humphries M, Sanford JR. A new approach to tuberculosis notification. $J$ Epidemiol Community Health 1984;38:331-4. 